\title{
Patients - Nurses - Providers: A Triad for Successful Use of a Daily ICU Rounding Checklist
}

\author{
Rahul Kashyap* and Katherine Heise
}

Critical Care Medicine, Mayo Clinic, Rochester, Minnesota, USA

\begin{abstract}
Daily checklists are shown to improve outcomes and adherence to quality measures in ICU care however completion of them consistently throughout institutions varies. Patients' loved ones are more present in the ICUs than in previous decades and can be a valuable resource for both information and use as a prompter for a daily checklist. In conjunction with the bedside nurse and health care providers, patients and their loved ones can add to the successful use of a daily ICU rounding checklist.
\end{abstract}

\section{Introduction}

The ICU is often a stressful and foreign place for patients and their loved ones. It is taxing related not only to the critical illness but also the uncertainty of the situation, surroundings, and routine. The health care staff members are accustomed to the daily routine of an ICU however we often lose sight of the fact that patients and their loved ones (for ease we will refer to the patient throughout the article meaning the patient or their advocate, loved ones, family members) are not.

\section{Body}

The healthcare providers are expected to provide quality care. Since mid -1920s the medical community has been publishing articles regarding quality care- how to measure it, and how to achieve it. In 1956 in the American Journal of Public Health, there was an article published describing methods how to evaluate and improve medical care that could be utilized in other medical programs as well [1]. Today, many ICU practices do daily rounds, many with interdisciplinary teams and collaborative teams. Holodinsky et al. found in their survey that $82 \%$ of the respondents did bedside rounds, $94 \%$ employed an open approach, $81 \%$ inter-professional, $86 \%$ reported a collaborative approach, and $48 \%$ using a tool to facilitate rounds [2]. While only about half reported using a rounding tool, it is shown that using a prompted checklist can improve some outcomes. A single-site study showed increased ventilator-free days, fewer days with empiric antibiotics and central lines, increased rates of both, deep vein thrombosis and peptic ulcer prophylaxis. When compared with the control group there was also lower risk-adjusted ICU and hospital mortality and lower ICU length of stay [3]. In a systematic review Lane et al. reported that there was strong evidence to do daily rounds in a standard location with defined roles and to minimize interruptions while focusing on daily goals [4]. The question then becomes, how do find ways to consistently complete a daily quality checklist?

In a survey of New England hospitals published in 2007, 32\% had unrestricted visiting hours [5]. This means that in a third of ICUs across New England family members are potentially present at the bedside 24 hours a day. Personal opinion about this fact may vary greatly but the fact remains that there is an ever present non-patient, non-staff population present in the ICU. This can either be deemed a distractor of care or embraced and utilized as a way to improve quality of care.

Quality care is difficult to define and quantify. It means different things to different people. One theme that tends to hold through conversations with patients is communication. Multiple tools are used throughout different ICUs to facilitate communication and dissemination of information to the patients and those at their bedside. Many facilities now use a patient portal that links to their electronic medical record so the patient is acutely aware of recent lab work, can often view notes written by providers, and even view radiographic images. Johns Hopkins WICU has a Family Involvement Menu that gives families things they can actively do with the patient to aide in their care [6].

Another component of quality care is the team approach. As healthcare is evolving we are discovering that a team approach to care is helpful as each team member brings their own perspective and knowledge to the situation. Jain et al. showed improved communication with multidisciplinary team approach [7]. The bedside team including nursing, the patient, and loved ones can be integral in providing information regarding history and current care. Extending this to the quality checklists that are routinely completed daily can lead to even better collaboration.

To facilitate a partnership between medical staff, patients, and their loved ones it is helpful to utilize a checklist which has been translated to nonmedical terminology. In this way patients or their loved ones can act as prompter throughout the day when to ensure all the quality measures are being addressed. Although it would be difficult to have them act as prompter during daily ICU rounds due to the varying schedules of both the patient and visitors, making available the translated checklist throughout the day can facilitate this role in collaboration with bedside nursing staff, patients, and providers.

The bedside nurse provides a pivotal role for this mode of communication. They are the consistent face to all of the teams including the patient. As the different teams come to the bedside each day the nurse is ever mindful of the plan. They are also the ones who

*Corresponding author: Rahul Kashyap, MBBS, Critical Care Medicine, Mayo Clinic Rochester, Minnesota, USA, Tel: 15072557196; E-mail: kashyap.rahul@mayo.edu

Received January 18, 2016; Accepted January 20, 2016; Published January 26,2016

Citation: Kashyap R, Heise K (2016) Patients - Nurses - Providers: A Triad for Successful Use of a Daily ICU Rounding Checklist. Adv Practice Nurs 2:104. doi:10.4172/2573-0347.1000104

Copyright: @ 2016 Kashyap R, et al., This is an open-access article distributed under the terms of the Creative Commons Attribution License, which permits unrestricted use, distribution, and reproduction in any medium, provided the original author and source are credited. 
Citation: Kashyap R, Heise K (2016) Patients - Nurses - Providers: A Triad for Successful Use of a Daily ICU Rounding Checklist. Adv Practice Nurs 2:104. doi:10.4172/2573-0347.1000104

Page 2 of 7

typically alert the provider to further needs, questions that may arise, and changes in the patient. With that, as the patient or loved one is utilizing the translated checklist they can collaborate to discuss the plan, the nurse can explain many of the questions, and contact the provider to update the patient on the plan when able.

\section{Conclusion}

This strategy can lead to increased communication between medical staff, the patient, and their loved ones. It is a way to not only educate the patient on ICU care, routines, and evidence based practice measures but it is also an opportunity to bring topics of discussion forward and ensure the quality checklist items are addressed each day.

\section{References}

1. Daily E, Morehead M (1956) A Method of Evaluating and Improving the Quality of Medical Care. American Journal of Public Health 848-854.
2. Holodinsky J, Hebert M, Zygun D, Rigal R, Berthelot S, et al. (2015) A Survey of Rounding Practices in Canadian Adult Intensive Care Units. PLOS ONE.

3. Weiss C, Moazed F, Mcevoy C, Singer B, Szleifer I, et al. (2011). Prompting Physicians to Address a Daily Checklist and Process of Care and Clinica Outcomes. American Journal of Respiratory and Critical Care Medicine 184: 680-686.

4. Lane D, Ferri M, Lemaire J, Mclaughlin K, Stelfox H (2013) A Systematic Review of Evidence-Informed Practices for Patient Care Rounds in the ICU* Critical Care Medicine 41: 2015-2029.

5. Lee MD, Friedenberg AS, Mukpo DH, Conray K, Palmisciano A, et al. (2007) Visiting hours policies in New England intensive care units: Strategies for improvement. Critical Care Medicine 35: 497-501.

6. (2016) Family Involvement Menu. (n.d.) Retrieved January 13.

7. Jain M, Miller L, Belt D, King D, Berwick DM (2006) Decline in ICU adverse events, nosocomial infections and cost through a quality improvement initiative focusing on teamwork and culture change. Quality and Safety in Health Care 15: $235-239$ 